\title{
Inductive Energy Harvesting From Current-Carrying Structures
}

\author{
S. W. Wright ${ }^{1}$, M. E. Kiziroglou ${ }^{1 *}$, S. Spasic ${ }^{2}$, N. Radosevic ${ }^{2}$ and E. M. Yeatman ${ }^{1 * *}$, \\ ${ }^{1}$ Imperial College London, SW7 2AZ, U.K. \\ ${ }^{2}$ Senis AG, 6340 Baar, Switzerland \\ * Senior Member, IEEE, ** Fellow, IEEE
}

Received 15 Apr 2019, revised 12 May 2019, accepted 18 May 2019, published .....2019, current version 18 May 2019. (Dates will be inserted by IEEE; "published" is the date the accepted preprint is posted on IEEE Xplore ${ }^{\circledR}$; "current version" is the date the typeset version is posted on Xplore ${ }^{\circledR}$ ).

\begin{abstract}
This paper introduces an inductive method for harvesting energy from current-carrying structures. Numerical simulation of a structural beam shows that the skin effect can lead to significant current concentration at edges, providing a five-fold power benefit at such locations, even at frequencies below $1 \mathrm{kHz}$. The use of a rectangular ferrite core can provide a $\times 4$ power density improvement. The adoption of funnel-like core shapes allows the reduction of core mass and coil frame size, leading to significant further power density enhancement. Magnetic field simulation and coil analysis demonstrate a power density increase of $\times 49$ by ferrite funnels, in comparison to a coreless coil. Experimental results demonstrate rectified power over $1 \mathrm{~mW}$ delivered to a storage capacitor, from a $40 \times 20 \times 2 \mathrm{~mm}$ core-and-coil, in the vicinity of a spatially distributed $20 \mathrm{~A}$ current at $800 \mathrm{~Hz}$. Rectification and impedance matching are studied experimentally using a voltage doubler circuit with input capacitor tuning to counteract the coil reactance. Experimental results from a spatially distributed $30 \mathrm{~A}$ current at $300 \mathrm{~Hz}$ and a $1: 7$ funnel core demonstrate power density of $36 \mu \mathrm{W} / \mathrm{g}\left(103 \mu \mathrm{W} / \mathrm{cm}^{3}\right)$, opening up the way to non-invasive inductive powering of systems in the vicinity of current-carrying structures.
\end{abstract}

Index Terms_-Inductive, energy harvesting, current carrier, power line, structures, aircraft.

\section{INTRODUCTION}

Energy harvesting can provide energy autonomy to wireless sensors, a key engineering requirement for emerging sensing technologies, provided that a suitable environmental source is available at the sensor vicinity. The state-of-art power availability exceeds $0.1 \mathrm{~mW} / \mathrm{cm}^{3}$ from most source types, which is adequate for supporting various wireless sensors at low duty cycle operation, e.g. 1\% [1]. However, narrow environmental condition requirements have limited the applicability of energy harvesting to bespoke sensor solutions. A high-priority objective is the development of energy harvesting power supplies, functional over a wider location range. This can be achieved by exploiting energy that is generally available within the internal range of a given sensing application group. For example, vibration of specific characteristics can be expected to be available in the environment of a terrestrial vehicle.

In some vehicles such as aircraft the return current path is common to multiple electrical systems and passes through structural elements, such as beams, rather than cables. This provides a varying magnetic field, available in a wide location range, which could potentially be exploited by inductive, or other magnetic force transducers to power wireless vehicle sensors.

Various methods of energy harvesting from power lines have been proposed in recent years. The employment of a piezoelectric beam with a permanent magnet printed at its tip was proposed in [2] for dual-core, bi-directional power lines. In [3], a similar method is followed, employing a Halbach array of permanent magnets to enhance the resulting magnetic force, and demonstrating $3 \mathrm{~mW} / \mathrm{cm}^{3}$, from a $10 \mathrm{~A} / 50 \mathrm{~Hz}$ power line. An electrostatic approach has been studied in [4] and [5], and a power output of $16 \mathrm{~mW}$ from a $0.1 \mathrm{~m}$ diameter, $0.2 \mathrm{~m}$ long tube, from a $60 \mathrm{kV} / 50 \mathrm{~Hz}$ power line is reported in [5]. Inductive coupling of a coil-and-core system to the magnetic field of power lines has also been studied [6-9]. A very interesting approach employing a bow-tie shaped core geometry has been reported in [9], showing a $\times 2.5$ increase of power in comparison with a cylindrical core implementation, and demonstrating $0.1 \mathrm{~mW} / \mathrm{cm}^{3}$ from a $11 \mu \mathrm{T}$ RMS, $50 \mathrm{~Hz}$ field. In another very promising implementation reported last year, magnetic core sheet geometries are used as flux guides, demonstrating a power enhancement as high as $\times 200$, and a power output of $1.5 \mathrm{~W}$ from a $100 \mathrm{~A}$ RMS power line and a credit-card sized coil-and-core device located on the side of the power line core [8]. Inductive power line harvesting on aircraft involves currents of variable frequency in the range up to $800 \mathrm{~Hz}$ and has been studied in [7], demonstrating around $0.6 \mathrm{~mW} / \mathrm{cm}^{3}$ using a closed-loop ferrite core around a 0.9 A RMS, $620 \mathrm{~Hz}$ power line.

In order to harvest energy from current-carrying structural elements, the spatial current distribution has to be taken into account. The use of mechanical structures with piezoelectric transducers is not advantageous for cases of varying frequency such as in aircraft, because of their requirement for resonant operation. In addition, an electrostatic approach is not possible due to the zero voltage of the return path. In this paper, the potential of inductive energy harvesting from the magnetic field of current-carrying structural elements is studied. The effects of frequency variation, current distribution and field - concentrating magnetic core geometries are evaluated by simulation and experimental results of power availability from spatially distributed currents using inductive coupling and a voltage 
doubler circuit are presented. The concept of inductive harvesting from a structural beam current is illustrated in Fig.1. The average power of a coil with loop area $\mathrm{A}, \mathrm{N}$ turns and resistance $\mathrm{R}$ will be:

$$
P_{A V}=\frac{V_{r m s}^{2}}{4 R}=\frac{V_{m}^{2}}{8 R}=\frac{\left(N \Phi_{m} \omega\right)^{2}}{8 R}
$$

where $V_{r m s}$ is the RMS open-circuit induced voltage, $V_{m}$ is the opencircuit voltage amplitude while $\Phi_{m}$ and $\omega$ are the amplitude and angular frequency of the magnetic flux through A.

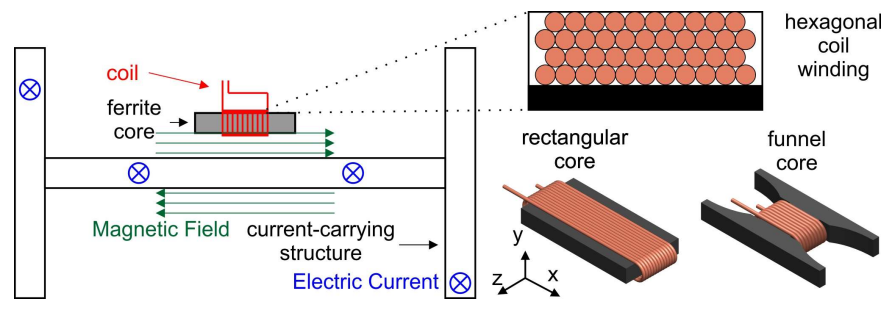

Fig. 1. The concept of inductive harvesting from a current-carrying structure. Coil winding and core shapes are illustrated on the right.

\section{THE SKIN EFFECT}

The current distribution in a structural beam was studied by finite element analysis using the Comsol software. The beam geometry corresponds to a H-shape structural rail beam of an aircraft, and the simulated material is aluminium. The web width and thickness are $140 \mathrm{~mm}$ and $4 \mathrm{~mm}$ respectively, while the two flanges are $55 \mathrm{~mm}$ and $40 \mathrm{~mm}$ wide with $4.5 \mathrm{~mm}$ and $3.8 \mathrm{~mm}$ thicknesses respectively. A beam length of $1 \mathrm{~m}$ was simulated using a current injector and sink located on the outer flat surfaces of each flange. The geometry and current density distribution results are shown in Fig. 2:Top. A total sinusoidal current of $1 \mathrm{~A}$ was simulated at $10 \mathrm{~Hz}$ (left) and $1 \mathrm{kHz}$ (right). The skin effect pushes current flow to the edges of the beam cross-section leading to significant current concentration at the tips of the flanges, at $1 \mathrm{kHz}$. This corresponds well with the analytically expected Al skin effect depth of $26 \mathrm{~mm}$ at $10 \mathrm{~Hz}$ and $2.6 \mathrm{~mm}$ at $1 \mathrm{kHz}$.

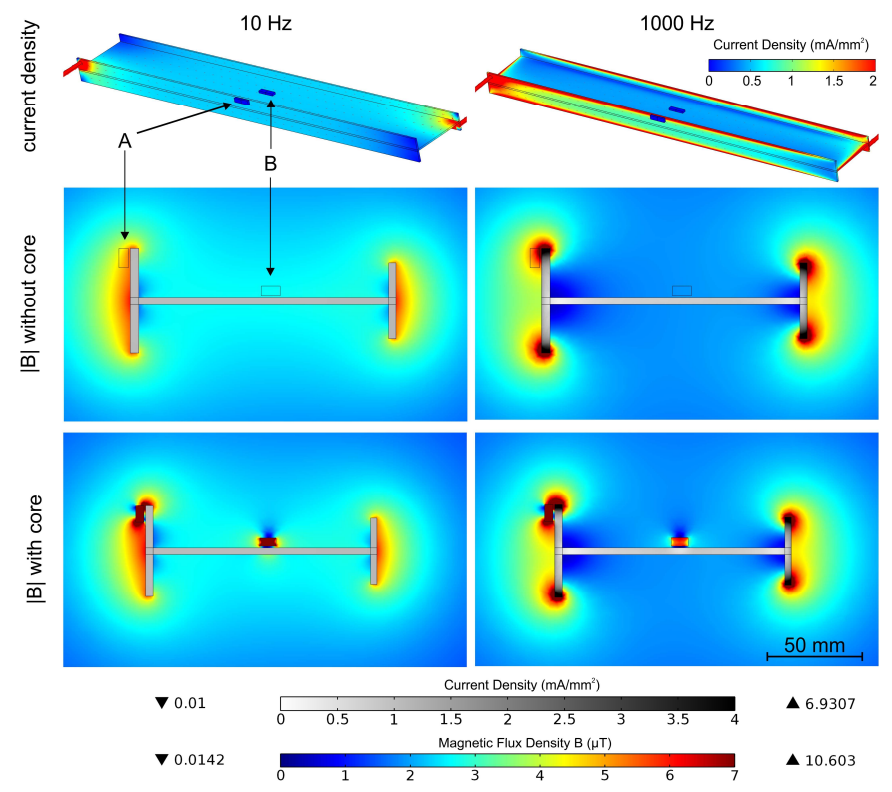

Fig. 2: Comsol simulation of current density $\mathrm{J}$ and magnetic flux density $B$ in a structural beam, for $10 \mathrm{~Hz}$ (Left) and $1 \mathrm{kHz}$ (Right), $1 \mathrm{~A}$ current.
Simulation results of the corresponding magnetic field around the beam are shown in the bottom four plots of Fig. 2, at the cross-section in the middle of the beam length. The current concentration at the beam flange edges results in significant field crowding around the flange tips at $1 \mathrm{kHz}$. This area is therefore beneficial for determining the optimal location of energy harvesting coil devices.

In order to quantify the skin effect benefit, the total flux available through a $40 \times 5 \mathrm{~mm}$ frame, with the long size perpendicular to the paper plane and the short axis in the horizontal direction in Fig.2, was calculated at the flange tip (location A) and the web center (location B) of the beam, by integrating the perpendicular magnetic flux density. The resulting flux amplitude as a function of frequency is plotted in Fig. 3 for a total current amplitude of 200 A. A $50 \%$ flux increase is observed for the beam tip location, and a $30 \%$ flux reduction at the beam center. The corresponding output power assuming a 2500 turn, $0.04 \mathrm{~mm}$ diameter $\mathrm{Cu}$ coreless coil is also plotted, demonstrating a $\times 5$ power difference between the two installation locations at $1 \mathrm{kHz}$. The corresponding volume, mass and resistance of the coil are $0.28 \mathrm{~cm}^{3}$, $2.5 \mathrm{~g}$ and $3 \mathrm{k} \Omega$ respectively, assuming a $20 \mathrm{~mm}$ winding length and a hexagonal close packing. The skin effect benefit can be calculated by comparison with the power scaling with $\mathrm{f}^{2}$ expected from Faraday's law, plotted as a dashed line in Fig. 3. A benefit of $125 \%$ at $1 \mathrm{kHz}$ and $64 \%$ at $300 \mathrm{~Hz}$ is observed for the side location.

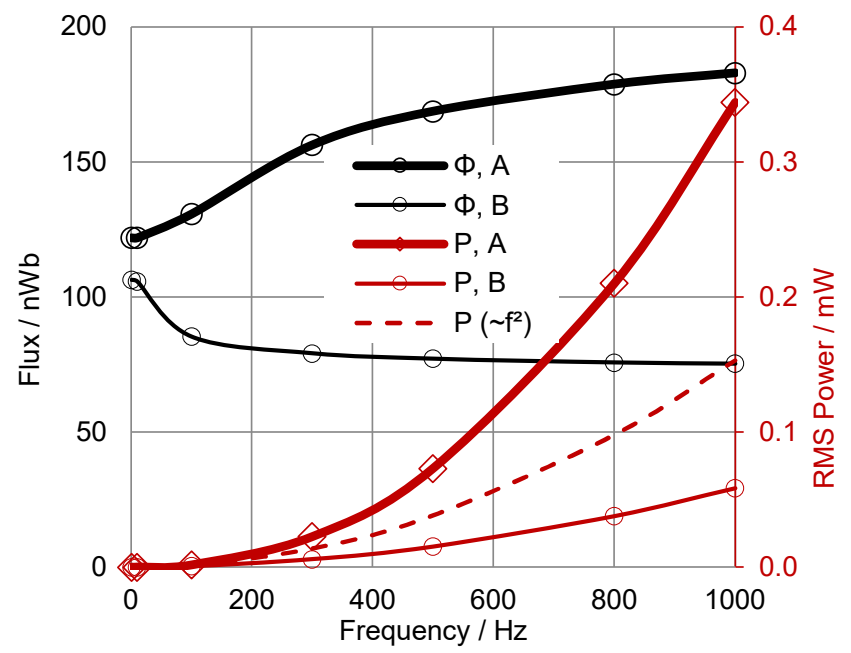

Fig. 3: Simulated flux and power from a $40 \times 5 \mathrm{~mm}$ frame at locations $A$ and B in Fig. 2, with a 2500 turn, $0.04 \mathrm{~mm}$ Cu wire, from a $200 \mathrm{~A}$ current.

\section{SOFT CORE GEOMETRIES}

In order to enhance the energy harvester power density, a soft magnetic material can be used inside the coil, to increase the magnetic flux density. This effect is illustrated in the simulation results of Fig. 2:Bottom, in which a rectangular $40 \times 5 \times 20 \mathrm{~mm}$ ferrite of $\mu_{\mathrm{r}}=200$ has been included. The employment of flux-concentrating funnel-like soft-core geometries could allow reduction of the coil cross-section while increasing the total flux. This can reduce the overall device size and mass as well as the coil resistance. To evaluate this effect, the performance of a coil-and-core device using a simple rectangular soft-core block, as shown in Fig. 2 is compared with that of a funnel-like soft-core structure. The two soft core geometries are illustrated in Fig. 4, with flux density distribution results corresponding to a $1 \mathrm{~A}, 1 \mathrm{kHz}$ structure current flow. 


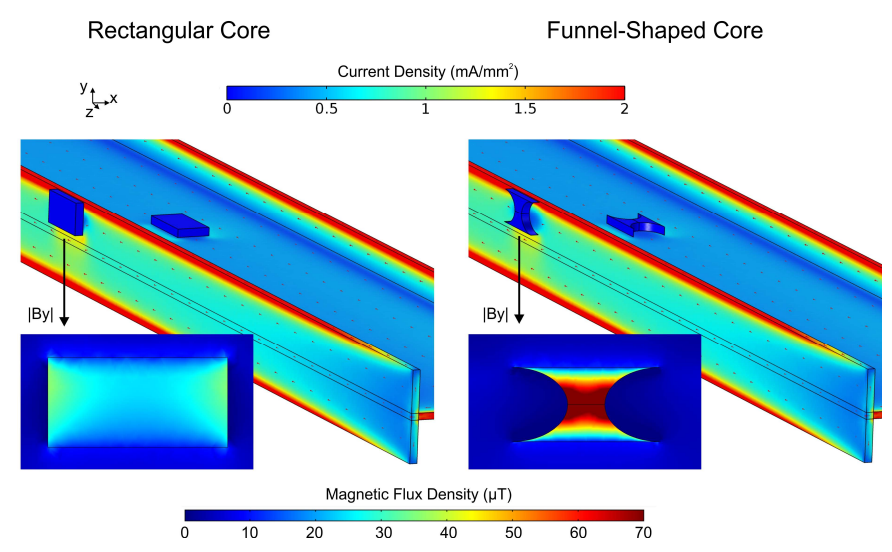

Fig. 4: Simulated current and flux density for two magnetic soft-core geometries, for a $1 \mathrm{~A}, 1 \mathrm{kHz}$ total structure current.

In order to study the soft-core effect to the power density of the energy harvester, the total flux available through the central $40 \times 5$ $\mathrm{mm}$ frame of the rectangular core and through the $10 \times 5 \mathrm{~mm}$ frame at the neck of the funnel-shaped core was calculated from the simulated perpendicular magnetic flux density. Results as a function of frequency are plotted in Fig. 5 for a total current amplitude of $200 \mathrm{~A}$, for the two core shapes as well as for a coreless $40 \times 5 \mathrm{~mm}$ frame. A milder flux dependence with frequency is observed due to the position of the coil frame which is lower than the flange top, where the largest current accumulation occurs. As in the previous section for the skin effect study, the corresponding output power assuming a 2500 turn, $0.04 \mathrm{~mm}$ diameter $\mathrm{Cu}$ coil with hexagonal close packing is also plotted in Fig. 5.

The six-fold flux increase provided by the rectangular core corresponds to $\mathrm{a} \times 36$ increase in power. The funnel structure provides a $\times 4.5$ flux increase but at a much smaller frame, which reduces the coil wire length. The corresponding coil resistance is $1 \mathrm{k} \Omega$, leading to the total power enhancement of $\times 60$ relative to the coreless case, observed in Fig. 5.

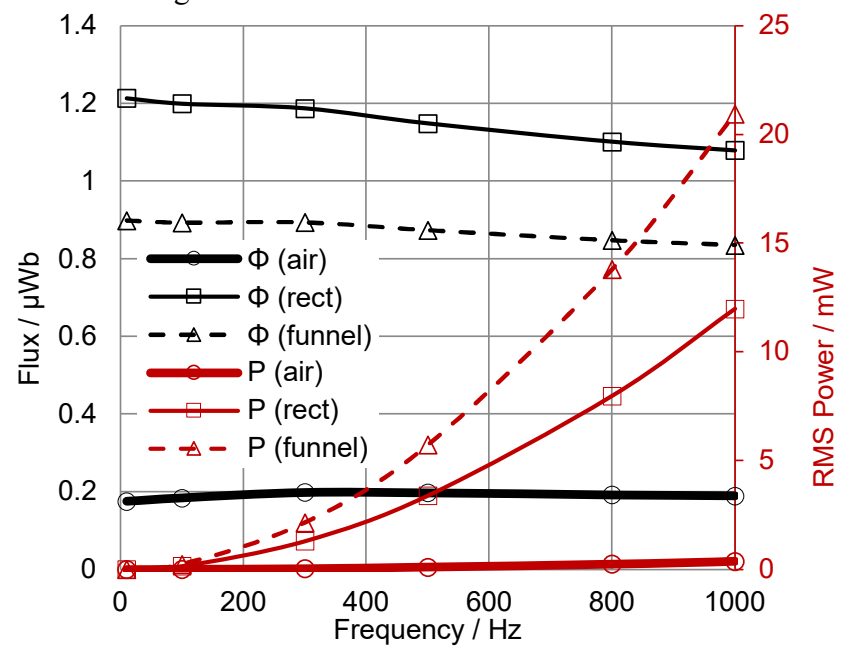

Fig. 5: Simulated flux and power output from a $40 \times 5 \mathrm{~mm}$ frame without a core, with a rectangular core and with a funnel-shaped core at its 10 x $5 \mathrm{~mm}$ neck frame. For the power output calculations, a 2500 turns, hexagonally packed $0.04 \mathrm{~mm}$ Cu wire coil is assumed. The structural current amplitude is $200 \mathrm{~A}$.
The benefit of funnel shaped geometries for different width narrowing ratios was evaluated by simulating different geometries and calculating the power output density, assuming the same 2500, $0.04 \mathrm{~mm}$ diameter $\mathrm{Cu}$ coil. In this study, a double length coil wire was assumed, both for resistance and weight calculations, to account for coil winding practical limitations. The power density was calculated by dividing the output power by the coil and core total mass. The results are shown in Fig 6 . The power density increases significantly with increasing top-to-neck width ratio. Magnetic saturation is not limiting the increase due to the low flux density range, up to structural current amplitudes as high as $1000 \mathrm{~A}$ for these geometries. In practice, the funneling ratio will be limited by mechanical fragility restrictions. For an 8:1 ratio a four-fold power density increase is achieved, in comparison with a rectangular core, reaching $0.18 \mathrm{~mW}$ per core-andcoil gram, or $0.96 \mathrm{~mW} / \mathrm{cm}^{3}$.

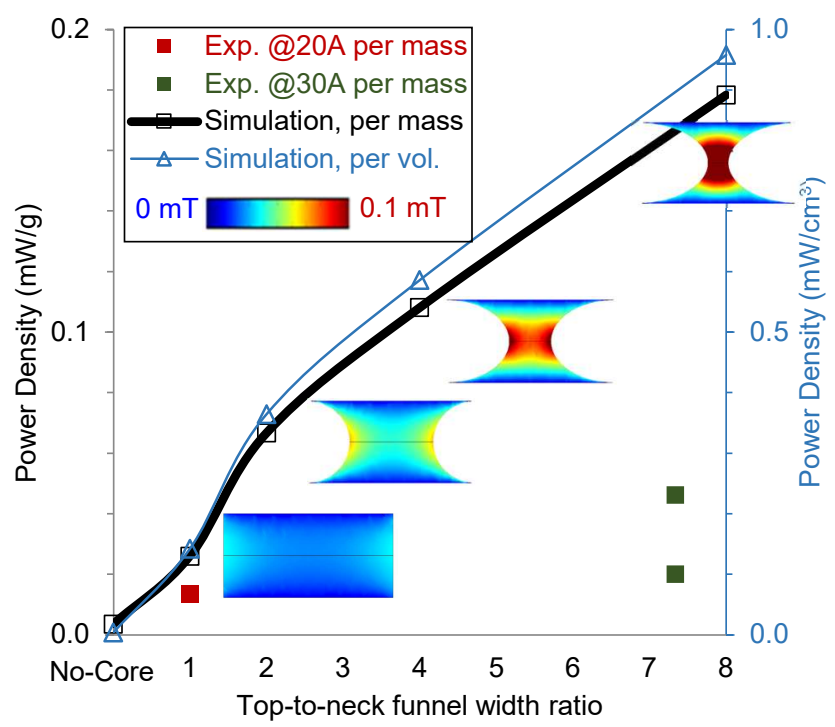

Fig. 6: Calculated power output density as a function of funnel core narrowing ratio, with 2500 turns of a $0.04 \mathrm{~mm} \mathrm{Cu}$ wire hexagonally distributed around the core neck, for a $300 \mathrm{~Hz}, 200 \mathrm{~A}$ structural current. The flux density distributions are also shown, for a $1 \mathrm{~A}$ structural current.

\section{EXPERIMENTAL RESULTS}

The possibility of energy harvesting from current carrying structures was evaluated experimentally using a 2500-turns, $40 \times 2 \mathrm{~mm}$ coil, and a $40 \times 2 \times 25 \mathrm{~mm}$ ferrite core. A spatiallydistributed current was emulated using a cable carrying a 1 A current of various frequencies. The cable was passed by the vicinity of the coil-and-core harvesting device 20 times in the same direction, forming loops of a large diameter (indicatively $1 \mathrm{~m}$ ), and creating a 20 A current distributed over a distance of $25 \mathrm{~mm}$. This corresponds approximately to the $0.2 \mathrm{~A} / \mathrm{mm}^{2}$ current distribution at the $25 \times 4.5$ $\mathrm{mm}$ beam flanges simulated in Sections II and III for a $200 \mathrm{~A}$ structural current at low frequencies, at which the skin effect is weak.

The coil is connected to a voltage doubler circuit for rectification and the output power is measured in a load resistor $\mathrm{R}_{\mathrm{L}}$, as shown in the inset of Fig. 7. Schottky diodes were selected to reduce losses. In order to maximize power transfer to the load, $\mathrm{R}_{\mathrm{L}}$ must be selected to be equal to the total equivalent impedance of the rest of the circuit. The first capacitor $\mathrm{C}_{\mathrm{t}}$ connected in series with the coil can be used to match and cancel the inductive part of the coil output resistance, 
thereby increasing the power output capability of the system. This effect was studied experimentally by measuring the output power as a function of $R_{L}$ for different $C_{t}$ and frequency values. The results are shown in Fig. 7. A maximum power output of $1 \mathrm{~mW}$ is obtained from the $800 \mathrm{~Hz}$ signal, at resonance and a $5.1 \mathrm{k} \Omega$ load. Taking into account the total core-coil mass, this corresponds to an experimental DC power density of $13.7 \mu \mathrm{W} / \mathrm{g}$, indicated as a red square in Fig. 6 . This result is $53 \%$ of the simulated $\mathrm{AC}$ power output for a $40 \times 5 \times 20 \mathrm{~mm}$ rectangular core. The lower experimentally demonstrated power density is attributed to core attribute deviations and to rectification losses. Experiments with a 1000-turn, 1:7 funnelling ratio ferrite core device, shown in the inset photo of Fig. 7, yielded $240 \mathrm{mV}$ and 360 $\mathrm{mV}$ open circuit voltage amplitude from $20 \mathrm{~A}$ and $30 \mathrm{~A}$ emulated distributed current respectively. These correspond to $20 \mu \mathrm{W} / \mathrm{g}$ (46 $\mu \mathrm{W} / \mathrm{cm}^{3}$ ) and $36 \mu \mathrm{W} / \mathrm{g}\left(106 \mu \mathrm{W} / \mathrm{cm}^{3}\right)$ respectively (green squares in Fig. 6). The $20 \mathrm{~A}$ and $30 \mathrm{~A}$ distributed current values used correspond to the total current in a single flange of the structure, without and with the skin effect at $300 \mathrm{~Hz}$. Precise fitting of simulations to the experimental conditions was avoided to maintain clarity in the simulation study.
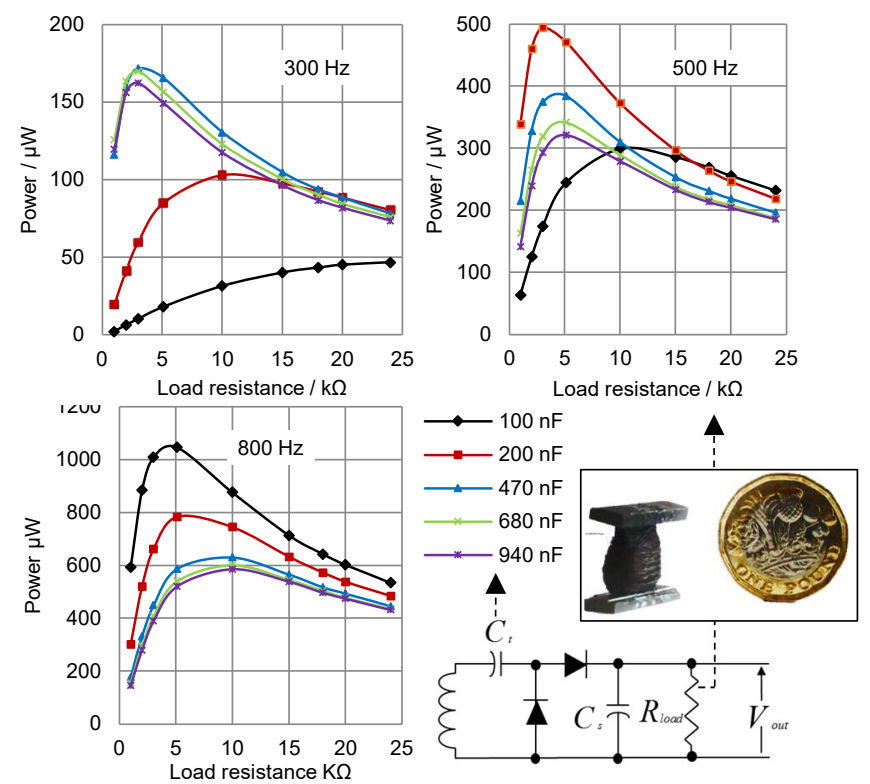

Fig. 7: Measurements from the Schottky voltage doubler circuit (bottom right) demonstrating harvesting power in the $0.1-1 \mathrm{~mW}$ range, reactance elimination by tuning $C_{t}$ and load matching for three different frequencies. A photograph of the 1:7 funneling device is also shown.

\section{CONCLUSIONS}

The possibility of inductive energy harvesting from the magnetic field of current-carrying structural beams was evaluated in this paper. The current distribution plays an important role and the skin effect can result in current accumulation at edges even at low frequencies $(10 \mathrm{~Hz}-1000 \mathrm{kHz})$. This can increase the output power if a beneficial installation location is selectable. For aircraft structural beams, simulations show a $\times 4$ and a $\times 5$ power benefit at edges, at $300 \mathrm{~Hz}$ and $1 \mathrm{kHz}$ respectively, in comparison to the beam centre. The employment of a ferrite core is shown to provide a power enhancement of $\times 36$, or $\times 4$ in power density (power per unit mass of the core-coil system), and $\times 6.5$ in power density per volume.

The power density can be further increased by adopting funnel-like core structures to guide more flux through smaller coil frames, reducing coil resistance as well as core mass. A power density improvement of over $\times 49$ is demonstrated by simulation from this method, in comparison to a coreless coil.

Experimental results using a rectangular core prototype demonstrate a rectified power density of $13.7 \mu \mathrm{W} / \mathrm{g}$ delivered to a storage capacitor, from a distributed $20 \mathrm{~A}$ current at $300 \mathrm{~Hz}$, and $84.4 \mu \mathrm{W} / \mathrm{g}$ at $800 \mathrm{~Hz}$. An impedance matching study shows that the maximum power transfer can be achieved by tuning the input capacitor of a voltage doubler circuit to eliminate the coil reactance, and match the load resistance to the resistance of the coil, in line with the theoretically expected $Z_{\text {load }}=Z_{\text {out }} *$ maximum power transfer point condition, where $Z_{\text {load }}$ and $Z_{\text {out }}$ * are the load impedance and the conjugate of the transducer output impedance.

Experimental results from a 1:7 flux funnelling device demonstrate $20 \mu \mathrm{W} / \mathrm{g}\left(46 \mu \mathrm{W} / \mathrm{cm}^{3}\right)$ and $36 \mu \mathrm{W} / \mathrm{g}\left(106 \mu \mathrm{W} / \mathrm{cm}^{3}\right)$ respectively from a distributed $20 \mathrm{~A}$ and $30 \mathrm{~A}$ current respectively at $300 \mathrm{~Hz}$. For comparison, the bow-tie shaped device results reported in [9] can be extrapolated to the simulated magnetic flux density and frequency of this work (spatial average $3.5 \mu \mathrm{T}$ RMS, $300 \mathrm{~Hz}$ ), giving $0.4 \mathrm{~mW} / \mathrm{cm}^{3}$. The flux guiding device in [8] provides around $16 \mathrm{~mW} / \mathrm{cm}^{3}$ from a $200 \mathrm{~A}$ RMS, $60 \mathrm{~Hz}$ current power line, while [7] reports $0.6 \mathrm{~mW} / \mathrm{cm}^{3}$ from 0.9 A RMS, $620 \mathrm{~Hz}$ and [6] reports $10 \mu \mathrm{W} / \mathrm{cm}^{3}$ from a household power line. Nevertheless, a direct figure-of-merit comparison among implementations is avoided due to substantial field source differences, especially given the varying distributed current in the structures studied in this work.

The employment of optimized funnel shaped geometries and tunable maximum power point tracking circuits is expected to enable inductive energy harvesting devices functional in various magnetic field sources, including both cable and structural current carriers. It is particularly suitable for powering sensors on aircraft or other vehicles, where structural beams are used as the return current path for variable frequency electrical power. In combination with other approaches such as flux guiding [8], it may also relax the installation location requirements, by extending the vicinity of operation.

\section{ACKNOWLEDGEMENT}

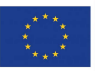

This project has received funding from the Clean Sky 2 Joint Undertaking under the European Union's Horizon 2020 research and innovation programme under grant agreement No 785495 and Project AMPWISE. This document reflects only the author's view and the Commission is not responsible for any use that may be made of the information it contains.

\section{REFERENCES}

[1] D. E. Boyle et al, "Energy Provision and Storage for Pervasive Computing," IEEE Pervasive Computing, vol. 15, no. 4, pp. 28-35, 2016.

[2] E. S. Leland et al, "A MEMS AC current sensor for residential and commercial electricity end-use monitoring," J. Micromech. Microeng., vol. 19 (9), 094018, 2009.

[3] W. He et al, "Energy harvesting from electric power lines employing the Halbach arrays," Review of Scientific Instruments, vol. 84, no. 10, p. 105004, 2013.

[4] F. Guo et al, "Energy harvesting devices for high voltage transmission line monitoring," 2011 IEEE Power and Energy Society General Meeting, pp. 1-8, 2011.

[5] X. Zhao et al, "Energy harvesting for a wireless-monitoring system of overhead highvoltage power lines," IET Gen.Transm. Distr. vol. 7, no. 2, pp. 101-107, 2013.

[6] O. Thorin, "Power Line Induction Energy Harvesting Powering Small Sensor Nodes," KTH Royal Institute of Technology, Sweden, 2016.

[7] T. Toh et al., "Inductive energy harvesting from variable frequency and amplitude aircraft power lines," J. Phys. Conf. Series., vol. 557, no. 1, p. 012095, 2014.

[8] R. M. White et al, "Atmospheric Sensors and Energy Harvesters on Overhead Power Lines," (in eng), Sensors (Basel), vol. 18, no. 1, Jan 32018.

[9] S. Yuan et al, Magnetic Field Energy Harvesting Under Overhead Power Lines, IEEE Transactions on Power Electronics, 2015, pp. 6191-6202. 